\title{
Real-world study of adding bevacizumab to chemotherapy for ovarian, tubal, and peritoneal cancer as front-line or relapse therapy (ROBOT): an observational cohort study
}

\author{
Pei-Ying $\mathrm{Wu}^{1}$, Ya-Min Cheng ${ }^{1}$, Meng-Ru Shen ${ }^{1}$, Yi-Chun Chen ${ }^{1}$, YU-FANG HUANG ${ }^{1}$, \\ and Cheng-Yang Chou ${ }^{1}$ \\ ${ }^{1}$ National Cheng Kung University Hospital, College of Medicine, National Cheng Kung \\ University
}

May 5, 2020

\begin{abstract}
Objective. This study aimed to determine the real-world, long-term prognostic impact and adverse effects (AEs) of bevacizumab (BEV) in Asian patients with ovarian/tubal/peritoneal cancers. Design Hospital-based observational cohort study. Setting Tertiary medical centre in Southern Taiwan. Population Women diagnosed with ovarian, tubal, peritoneal cancer. Methods. We retrospectively reviewed the medical records of consecutive patients on front-line chemotherapy with or without BEV (Cohort 1) and those who relapsed following chemotherapy and/or BEV (Cohort 2) between 2011 and 2018. Main outcome measures Patient clinicopathological characteristics, BEV dosages, clinical outcomes, survivals, and AEs were analysed. Hazard ratios for disease progression and death were analysed using a Cox model. Results. Benefits of BEV used throughout triweekly, in terms of improved progression-free survival (PFS) and overall survival (OS), were observed at a dosage of $7.5-15 \mathrm{mg} / \mathrm{kg} / \mathrm{cycle}$ among advanced-stage Cohort 1 patients. A progression-free interval of $<6$ months was the strongest predictor of disease progression and death in advanced-stage patients. BEV throughout and optimal cytoreduction were independent predictors of reduced disease progression. Histology was not a prognostic predictor. BEV resulted in improved OS in Cohort 2 patients, especially in the platinum-sensitive subgroup. Most patients had a front-line BEV dosage $<10 \mathrm{mg} / \mathrm{kg}$ per cycle with $<10$ treatment cycles. Low rates and grades of BEV-related AEs were observed. Conclusions BEV used throughout effectively extended PFS and OS in advanced-stage patients. Patients with platinum-sensitive carcinoma, treated with BEV, had a significant improvement in OS and extended PFS. Therefore, BEV can safely be added to chemotherapy for ovarian/tubal/peritoneal cancers.
\end{abstract}

\section{Funding}

This clinical research received funding from the headquarters of the University Advancement at the National Cheng Kung University, which is sponsored by the Ministry of Education, Taiwan, ROC (to YFH). None of the funding sources were involved in collecting, analysing or interpreting data, writing the article or deciding to submit for publication.

Keywords: ovarian neoplasms, fallopian tube neoplasms, bevacizumab, vascular endothelial growth factor, progression, survival, drug-related side effects and adverse reactions

\section{Tweetable abstract}

Advanced-stage patients with ovarian cancer treated with front-line bevacizumab throughout had improved overall survivals.

\section{Introduction}


Epithelial ovarian cancer (EOC) is the leading cause of death among gynaecological cancers in many countries. ${ }^{1-3}$ Most patients with chemotherapy-sensitive disease develop chemotherapy-resistant relapse after undergoing several lines of therapy. Maintenance therapy with targeted agents following primary cytoreductive surgery and chemotherapy to extend the progression-free interval (PFI) is promising for improving EOC prognosis. ${ }^{4,5}$ Bevacizumab (BEV) can improve overall response rates in front-line or relapse therapy settings. ${ }^{5,6}$ Front-line chemotherapy with BEV throughout improved progression-free survival (PFS) in the GOG-218 ${ }^{7}$ and ICON7trials ${ }^{8}$ and prolonged overall survival (OS) in GOG-218 stage IV and ICON7-defined high-risk patients. ${ }^{9,10}$ The reductions in the risk of progression or death by adding BEV to chemotherapy were observed in platinum-sensitive recurrent EOC in the OCEANS ${ }^{11}$ and GOG-213 trials, ${ }^{12}$ respectively. A $52 \%$ reduction in the risk of progression or death was seen in platinum-resistant recurrent EOC in AURELIA. ${ }^{13}$ However, OS was not significantly extended. ${ }^{9,11-13}$

BEV-related adverse effects (AEs) are well understood. ${ }^{7,8,11-14}$ Patients treated with BEV experienced a higher incidence of hypertension, proteinuria, gastro-intestinal (GI) and non-central nervous system (nonCNS) bleeding events, and thromboembolism (TE) than those not treated with BEV.

Based on these large clinical trials, BEV is used as the standard of care in EOC patients in many countries. However, the medical cost of adding BEV to chemotherapy may make this treatment unaffordable for EOC patients if it is not covered by the national health insurance (NHI). Moreover, the disease status in real-world patients is much more complex than that in clinical trials. Some real-world investigations have focused on the impact on PFS and AEs when BEV is added to front-line treatment. ${ }^{15-17}$ More real-world data to compare BEV effects on prognosis in recurrent EOCs or AEs between patients with or without prior BEV treatment are needed to assess cost-effectiveness. ${ }^{18-20}$

Therefore, our study focused on EOC/tubal cancer (TC)/primary peritoneal cancer (PPC) patients who underwent front-line paclitaxel-carboplatin chemotherapy \pm BEV or those with recurrent/persistent disease who underwent BEV \pm chemotherapy. Our primary aim was to describe the correlation between clinicopathological factors and physician-patient shared-decision making (SDM), as well as the survival benefits of adding BEV to front-line chemotherapy. Secondarily, we aimed to determine the safety of combination regimens of BEV plus chemotherapy, with or without prior BEV use. We aimed to identify advanced-stage or ICON7-defined high-risk patients who may have a more favourable outcome in the front-line setting and predict which subgroup has a better prognosis after adding BEV.

\section{METHODS}

\section{Participants}

The clinical research protocol was approved by the National Cheng Kung University Hospital Institutional Review Board (Protocol No. A-ER-108-119). The study was performed in accordance with the Declaration of Helsinki. The requirement for informed consent was waived due to the retrospective nature of the study. The study flow chart regarding patient inclusion is illustrated in Figure S1 . Patients with histologically proven EOC/TC/PPC were considered eligible if they received front-line platinum-based chemotherapy as preoperative (neoadjuvant) or postoperative (adjuvant) therapy (Cohort 1) or if their relapsed diseases were treated with BEV \pm any chemotherapy (Cohort 2) between January 1, 2011 and August 31, 2019.

Medical records were reviewed for patients' clinical characteristics, treatment-related AEs, and treatment outcomes. Patients without postoperative front-line chemotherapy owing to any reason and those who had non-paclitaxel-platinum doublet were excluded. Cancer stage was determined according to the criteria of the International Federation of Gynecology and Obstetrics (FIGO). Residual nodules $<1 \mathrm{~cm}$ and [?]1 cm were categorised as "optimal" and "suboptimal," respectively. Cancer progression was defined based on the objective Response Evaluation Criteria in Solid Tumors 1.1 or the Gynecologic Cancer InterGroup (GCIG) definition. The last record was retrieved on August 31, 2019. AE severity was graded according to the Common Terminology Criteria for Adverse Events, version 4.03. OS was calculated based on the diagnosis date. PFS and PFI were determined using the date of last contact or progression after front-line chemotherapy. PFI $<6$ months $(\mathrm{m})$ and [?]6 m were categorized as "platinum-resistant" and "platinum- 
sensitive," respectively. "PFS2-PFS" was calculated based on the date of the first relapse or persistence to the next progression or death.

\section{Statistical analysis}

Data were analysed using SPSS (version 19.0; SPSS Inc., Chicago, IL, USA). Interval variables are presented as means +- standard deviations. Differences between groups were tested using the Mann-Whitney U test. Frequency distributions between categorical variables were compared using Pearson $X^{2}$ or Fisher's exact tests. Survival was estimated using the Kaplan-Meier method and compared using log-rank tests. $\mathrm{P}<0.05$ (two-sided) was considered statistically significant. Cox proportional hazards models were used to estimate hazard ratios (HRs) and confidence intervals (CIs). Possible confounders were included in multivariate analyses. The independent effect of BEV use on survival and disease progression was analysed in the multivariate analysis.

\section{RESULTS}

\section{Patient demographics}

Cohort 1 patients

Newly diagnosed patients $(\mathrm{n}=381)$ aged $21-88$ years $(\mathrm{y})$ (mean, $53.6+-11.4$ y) were enrolled. Only 37 patients were aged $>70$ y. Most patients $(n=359,94.2 \%)$ were diagnosed with EOC; eight (2.1\%) were diagnosed with TC and $14(3.7 \%)$ with PPC. Of these, $77(20.2 \%)$ received postoperative paclitaxel-carboplatin doublet plus BEV. A total of 147 (38.6\%) were classified as having FIGO stages I-II and 234 (61.4\%) as having stages III-IV; 116 (30.4\%) patients were in the ICON7-defined high-risk group. More than half of the patients $(n=203,53.3 \%)$ had serous histology, 108 (28.3\%) had clear cell carcinoma (CCC) histology, 39 $(10.2 \%)$ had endometrioid histology, and 20 (5.2\%) had mucinous histology. The median follow-up period was $33.7 \mathrm{~m}$ (range 1.7-99.9 m). BEV dosage was 7.5-15.1 (mean, $9.2+-1.5$ ) $\mathrm{mg} / \mathrm{kg}$, and number of treatment cycles was $1-17$ (mean $7.9+-5.0$ ). The dosage was $<10 \mathrm{mg} / \mathrm{kg} /$ cycle in $71.4 \%$ of patients, and $<10$ cycles were used in $76.6 \%$ of patients. BEV dosages were similar between stage III-IV $(\mathrm{n}=67)$ and ICON7-defined high-risk patients $(\mathrm{n}=37)$ (mean, $9.3+-1.6$ and $9.3+-1.8 \mathrm{mg} / \mathrm{kg}$, respectively). There was no difference in the number of treatment cycles between these groups (mean, $7.9+-5.0$ and $7.9+-4.6$ cycles, respectively). During follow-up, 45 patients $(58.4 \%)$ in the chemotherapy plus BEV group developed progressive disease, and $22(28.6 \%)$ died; 156 patients $(51.3 \%)$ in the chemotherapy alone group had progressive disease, and 114 patients $(37.5 \%)$ died.

Relationships between whether BEV was added to chemotherapy and clinicopathological factors are presented in Table 1 . A significantly higher proportion of women with advanced stage, serous histology, preoperative chemotherapy, residual tumour size [?]1 cm, and triweekly delivery of postoperative chemotherapy received BEV.

Cohort 2 patients

Sixty-five patients with relapsed or persistent disease (mean age, 52.5 +- 11.0 y; range, 18-74 y) were enrolled. Patients' baseline characteristics are shown in Table S1. Of those treated with various chemotherapy regimens, $39(60.0 \%)$ received adjuvant platinum-based chemotherapy and $22(33.8 \%)$ had prior BEV use. BEV dosage was 7.0-12.6 (mean, 9.5 +- 1.3) mg/kg and 7.9-11.6 (mean, $10.2+-1.1$ ) mg/kg for patients with the first and [?]2 relapses, respectively; the number of treatment cycles was 1-22 (mean 5.3 +- 3.1) and 2-19 (mean 6.5 +- 4.4), respectively. The median follow-up period was $3.6 \mathrm{~m}$ (range $0.1-37.3 \mathrm{~m}$ ). Most patients $(\mathrm{n}=60,92.3 \%)$ were diagnosed with EOC; $2(3.1 \%)$ and $3(4.6 \%)$ were diagnosed with TC and PPC, respectively. Thirty-nine patients (60.0\%) had serous histology, 10 (15.4\%) had CCC histology, 6 (9.2\%) had endometrioid histology, and 4 (6.2\%) had mucinous histology. During follow-up, 39 patients (60.0\%) developed progressive disease, and 29 (44.86\%) died.

Relationships between PFI $<6$ or [?]6 $\mathrm{m}$ and clinicopathological factors are presented in Table S1 . No statistical difference between PFI of $6 \mathrm{~m}$ and stage, histology, tumour grade, residual tumour size during 
primary surgery, or next disease progression was observed.

\section{Clinical outcomes}

Cohort 1 patients with advanced-stage disease

Survival curves for 234 patients stratified by means of BEV added to chemotherapy or chemotherapy alone are illustrated in Figure 1. The median PFS duration was greater in the subgroup with chemotherapy plus BEV than with chemotherapy alone (11.6 $\mathrm{m}$ versus $9.3 \mathrm{~m}, \mathrm{P}=0.325$; HR 0.84, 95\% CI, 0.6-1.19) among advanced-stage patients (Figure 1A), similar to that found among ICON7-defined high-risk patients (10.5 $\mathrm{m}$ versus $6.0 \mathrm{~m}, \mathrm{P}=0.035$; HR 0.62, 95\% CI, 0.39-0.97) (Figure 1B). The median OS was not achieved in the subgroup with BEV and was $43.7 \mathrm{~m}$ in those without $\mathrm{BEV}(\mathrm{P}=0.123$; HR 0.69, 95\% CI, 0.43-1.11) among advanced-stage patients (Figure 1A); similar results were seen among ICON7-defined high-risk patients (not reached versus $34.7 \mathrm{~m}, \mathrm{P}=0.101$; HR 0.61, 95\% CI, 0.33-1.10) (Figure 1B) . Compared to patients with chemotherapy alone, among ICON7-defined high-risk patients, patients with BEV throughout had a significantly greater PFS $(17.6 \mathrm{~m} ; \mathrm{P}=0.005$; HR 0.39, 95\% CI, 0.20-0.75) and OS (not reached; $\mathrm{P}=$ 0.010; HR 0.22, 95\% CI, 0.07-0.72) (Figure 1D). However, among advanced-stage patients, patients with BEV throughout had a significantly greater OS (not reached; P $=0.030$; HR 0.41, 95\% CI, 0.18-0.93), but not PFS, than those with chemotherapy alone (Figure 1C).

Advanced-stage patients treated with chemotherapy plus BEV in the subgroup of PFI $<6 \mathrm{~m}$ had a significantly greater PFS (P = 0.001), but not OS (Figure S2). No statistical difference in the impacts of BEV on PFS or OS between clear cell and serous histology was observed in the early or advanced stage (Figure S3) .

Cohort 2 with relapsed or persistent disease

The survival curves stratified by PFI of 6 and $12 \mathrm{~m}$ are illustrated inFigure 2 . Patients with PFI [?]6 m after primary therapy had a significantly better OS and PFS than those with PFI $<6 \mathrm{~m}(P<0.001$ and $P<$ 0.001, respectively) (Figure 2A ). Patients with a longer PFI had more favourable survival. Patients with PFI [?]12 $\mathrm{m}$ had greater OS and PFS than those with PFI $<6 \mathrm{~m}(P<0.001$ and $P<0.001$, respectively $)$ (Figure 2B) .

\section{Univariate and multivariate analyses}

In the univariate analysis, residual nodules [?]1 $\mathrm{cm}$ and PFI $<6 \mathrm{~m}$ were significantly associated with a high risk of death and disease progression in Cohort 1 patients with advanced disease (Table 2). Age [?]53 years was associated with a high risk of death due to cancer. In a multivariate-adjusted model, PFI $<6 \mathrm{~m}$ was the strongest predictor of disease progression (HR 27.78, 95\% CI 15.87-47.62) and death (HR 5.92, 95\% CI $3.82-9.17)$. BEV throughout and optimal cytoreduction in the front-line setting were independent predictors of reduced cancer progression (HR 0.43, 95\% CI 0.25-0.73, and HR 0.63, 95\% CI 0.44-0.90, respectively). Although BEV throughout was a predictor of cancer death, its impact was influenced by confounders in the multivariate analysis.

By cox proportional analysis in Cohort 2 patients, those with PFI of [?]6 $\mathrm{m}$ after primary therapy had significantly lower risks of death (HR $0.22,95 \%$ CI $0.10-0.50$ ) and next progression (HR $0.17,95 \%$ CI 0.08-0.39) than those with PFI $<6 \mathrm{~m}$.

\section{Safety assessment}

In total, 142 (77 Cohort 1 and 65 Cohort 2) patients were assessed for AEs (Table S2 ). Most AEs were mild ([?] grade 2), treatable, and reversible. Commonly observed AEs [?] grade 3 included neutropenia $(\mathrm{n}=44$, $31.0 \%)$, febrile neutropenia $(\mathrm{n}=12,8.4 \%)$, anaemia $(\mathrm{n}=6,4.2 \%)$, diarrhoea $(\mathrm{n}=2,1.4 \%)$, and anorexia $(\mathrm{n}=1$, $0.7 \%)$. BEV-associated AEs were not observed. All cases of non-CNS bleeding $(\mathrm{n}=26,3.5 \%)$, hypertension $(\mathrm{n}=21,14.8 \%)$, proteinuria $(\mathrm{n}=13,9.2 \%)$, or venous thromboembolic events $(\mathrm{n}=3,2.1 \%)$ were rare and treatable in patients with or without prior BEV use. 


\section{DISCUSSION}

\section{Main findings}

We found improved PFS as a benefit of BEV throughout triweekly in the dosage range of $7.5-15 \mathrm{mg} / \mathrm{kg}$, which is consistent with results of independent phase 3 trials assessing front-line therapy ${ }^{7,8}$ and real-world data. ${ }^{15-17} \mathrm{PFI}<6 \mathrm{~m}$ independently predicted shorter PFS and OS, but BEV may reduce platinum-resistant relapse; these findings are in concordance with the prospective single-arm results in JGOG3022. ${ }^{17}$

\section{Strengths and limitations}

To our knowledge, this is the first real-world study to provide data on improved OS as an advantage of BEV throughout, relative to chemotherapy alone, in front-line therapy in advanced-stage EOC patients and the ICON7-defined high-risk subgroup.

The prognostic factors, e.g. advanced stage, histology, pre- and post-operative chemotherapy, and residual tumour size, were adjusted for during multivariate analysis, but expert-patient SDM regarding BEV utilization may possibly be influenced by choices of post-progression therapy or socio-economic factors which were not included in our analysis.

There are some other limitations to our study. BEV and BRCA/ homologous recombination deficient (HRD) testing has not yet been covered by our NHI. Hence, we are unable to provide real-world data regarding BEV in patients with BRCA mutation/HRD. Although most AEs were mild and treatable, the number of patients treated with BEV after prior BEV is too small to draw a conclusion in our relapsed cohort. Further research should be conducted to clarify these questions.

\section{Interpretation}

The GCIG considered PFS as the primary endpoint in front-line therapy for ovarian cancer, and not OS, because of the confounding effects of post-progression therapy on OS. ${ }^{7,21}$ Advances in various postprogression therapies, such as BEV, ${ }^{11-14,18}$ trientine, ${ }^{22}$ and poly(adenosine diphosphate [ADP]-ribose) polymerase (PARP) inhibitor, ${ }^{4,23-25}$ may help extend patients' lives. We provided evidence while we observed a longer median OS in the BEV throughout group compared to that in GOG-218 and ICON7, even in patients who received only chemotherapy. When adjusted by multivariate analysis, our real-world findings corresponded to the GCIG consensus and emphasized the role of BEV throughout in PFS prolongation. This phenomenon is observed in the improved OS after using BEV in relapsed EOC, specifically in the platinum-sensitive subgroup.

A significantly improved PFS when adding olaparib after a response to chemotherapy plus BEV has occurred and a higher incidence of reversible grade [?] 3 haematologic AEs were reported in the PAOLA-1 trial. ${ }^{26}$ Tewari et al. reported the reductions in risk of death for BRCA1/2-mutated or non-BRCA1/2 HRD carcinomas when compared to the wild type. ${ }^{9}$ However, BRCA/HRD testing was not predictive of BEV activity. Our patients had a shorter median PFS than that of the non-Olaparib arm in PAOLA-1 trial ${ }^{26}$ or those in real-world studies. ${ }^{15-17}$ The reason for these discrepancies may be multifactorial, including different study designs, shorter maintenance duration or lower dosage of BEV, more complex disease, or reimbursement issues in the real-world setting.

Platinum resistance is an indicator of poor prognosis, and its involved mechanism is complex. ${ }^{27-29}$ Lee et al. reported that the effectiveness of BEV-included chemotherapy was feasible but varied according to the chemotherapy partner in platinum-resistant EOC. ${ }^{20}$ A real-world small study showed PFS benefits of early BEV-added chemotherapy, but insignificant survival differences between platinum-resistant and -sensitive recurrent EOC. ${ }^{30}$ However, we provided more favourable oncologic outcomes of BEV-added relapse therapy in platinum-sensitive patients. Furthermore, CCC has been thought of as a platinum-resistant malignancy. The histological distribution of EOC in Asia is quite different from that in the West. Serous, endometrioid, and CCC histology constituted $40-50 \%, 15-20 \%$, and 15-20\%, respectively, of EOC in Taiwan from 20002008. ${ }^{31}$ Komiyama et al. reported that BEV-added front-line therapy was effective for advanced-stage clear 
cell carcinoma. ${ }^{17}$ However, the lack of benefits of BEV on clinical outcomes across different histologies in our study is similar to findings recorded in the ICON7 final results. ${ }^{10}$ Therefore, BEV could be applied regardless of histology in either an early or advanced stage.

Haematologic AEs were the most common in BEV-included trials in EOC, $, 8,11-14$ while similar results were observed in our study. Its safety was also promising in our relapse therapy. The cumulative incidences of hypertension and proteinuria were associated with median cumulative BEV dosages ${ }^{32}$ or the treatment durations $^{17}$ in Asian women. However, our patients had less BEV-specific incidences and lower grades of AEs. This may be related to lower dosages, or fewer treatment cycles in most patients, which were in concordance with lower incidence rates of hypertension, proteinuria, GI, or non-CNS bleeding events in the BEV throughout arm in ICON7 than that in GOG-218. ${ }^{7,8}$

\section{Conclusion}

Adding BEV to traditional front-line or relapse therapy was safe in EOC. This strategy as maintenance therapy was effective in extending PFS and OS in advanced-stage patients. Platinum-sensitivity was the strongest prognostic factor. Platinum-sensitive relapsed EOC patients treated with BEV-added chemotherapy had a significant improvement in OS and had a longer duration in progressing to the next progression.

Ethics statement The Institutional Review Board of National Cheng Kung University Hospital approved the study protocol on 29 April 2019 (ref. no. B-ER-101-318). The study was performed in accordance with the Declaration of Helsinki. The requirement for informed consent was waived due to the retrospective nature of the study.

Disclosure of interests YFH got grants from the headquarters of the University Advancement at the National Cheng Kung University, which is sponsored by the Ministry of Education, Taiwan, ROC, during the conduct of the study. None of the funding sources were involved in collecting, analysing or interpreting data, writing the article or deciding to submit for publication. The other authors declare no competing interests. Full disclosure of interests is available to view online as supporting information.

Acknowledgments This research was carried out and administratively supported by NCKUH, College of Medicine, National Cheng Kung University. We acknowledge the efforts made by our nursing practitioners (Yu Hsiao Tai, Ya-Ting Chen, Ya-Ting Hu, Yueh-Chin Li, Shu-Jyun Wang, and Ya-Han Tsai) in assessing adverse effects related to chemotherapy or target therapies.

\section{Contribution to authorship}

PYW, YMC, YFH and CYC conceived and designed the study. PYW, YMC, MRS, YFH, and CYC conducted the study. PYW collected the data. YCC, and YFH performed the statistical analysis. PYW, YFH, and CYC interpreted data for the work. PYW, YMC, MRS, YCC, YFH, and CYC drafted the manuscript. YFH and CYC revised the manuscript. All authors reviewed and approved the final manuscript and have agreed to be accountable for all aspects of the work.

\section{Funding}

This clinical research received funding from the headquarters of the University Advancement at the National Cheng Kung University, which is sponsored by the Ministry of Education, Taiwan, ROC. None of the funding sources were involved in collecting, analysing or interpreting data, writing the article or deciding to submit for publication.

\section{Supporting information}

Additional supporting information may be found online in the Supporting Information section at the end of the article.

Table S1. Clinico-pathological characteristics of patients with recurrent or persistent disease $(\mathrm{N}=65)$ 
Table S2. Adverse effects and grades in ovarian/tubal/peritoneal patients treated with bevacizumab plus chemotherapy $(\mathrm{N}=142)$.

Figure S1. Study flow chart

(A) Cohort 1 patients underwent first-line paclitaxel-carboplatin chemotherapy with or without bevacizumab (BEV).(B) Cohort 2 patients with relapsed or persistent disease treated with BEV +- chemotherapy. Patients were also categorized as groups with progression-free interval (PFI) $<6$ and [?] $6 \mathrm{~m}$.

Figure S2. Progression-free and overall survival between paclitaxel-carboplatin chemotherapy (Chemo) with and without bevacizumab (BEV) in stage III-IV patients undergoing first-line therapy were tested using the log-rank test (A) in the progression-free interval (PFI) [?]6 m subgroup and (B) in the PFI $<6 \mathrm{~m}$ subgroup.

Figure S3. Progression-free and overall survival curves stratified by the use of bevacizumab in subgroups of serous and clear cell histology in an early and advanced stage, tested by the log-rank test. (A) Early-stage clear cell carcinoma group, (B)early-stage serous carcinoma group, (C) advanced-stage clear cell carcinoma group, and (D) advanced-stage serous carcinoma group.

\section{REFERENCES}

1. Siegel RL, Miller KD, Jemal A. Cancer statistics, 2018. CA Cancer J Clin 2018;68:7-30.

2. Ferlay J, Colombet M, Soerjomataram I, Mathers C, Parkin DM, Pineros M et al. Estimating the global cancer incidence and mortality in 2018: GLOBOCAN sources and methods. Int $J$ Cancer 2019;144:1941-53. doi:10.1002/ijc.31937

3. Chang LC, Huang CF, Lai MS, Shen LJ, Wu FL, Cheng WF. Prognostic factors in epithelial ovarian cancer: A population-based study.PLoS One 2018;13: e0194993.

4. Franzese E, Centonze S, Diana A, Carlino F, Guerrera LP, Di Napoli M, et al. PARP inhibitors in ovarian cancer. Cancer Treat Rev2019;73:1-9. doi:10.1016/j.ctrv.2018.12.002

5. Jiang Y, Sun X, Kong B, Jiang J. Antiangiogenesis therapy in ovarian cancer patients: An updated meta-analysis for 15 randomized controlled trials. Medicine (Baltimore) 2018;97:e11920. doi:10.1097/MD.0000000000011920

6. Wu YS, Shui L, Shen D, Chen X. Bevacizumab combined with chemotherapy for ovarian cancer: an updated systematic review and meta-analysis of randomized controlled trials. Oncotarget 2017;8:1070313.

7. Burger RA, Brady MF, Bookman MA, Fleming GF, Monk BJ, Huang H et al. Incorporation of bevacizumab in the primary treatment of ovarian cancer. $N$ Engl J Med 2011;365:2473-83. doi:10.1056/NEJMoa1104390

8. Perren TJ, Swart AM, Pfisterer J, Ledermann JA, Pujade-Lauraine E, Kristensen G et al. A phase 3 trial of bevacizumab in ovarian cancer [published correction appears in N Engl J Med. 2012 Jan 19;366(3):284]. N Engl J Med 2011;365: 2484-96. doi:10.1056/NEJMoa1103799

9. Tewari KS, Burger RA, Enserro D, Norquist BM, Swisher EM, Brady MF et al. Final Overall Survival of a Randomized Trial of Bevacizumab for Primary Treatment of Ovarian Cancer. J Clin Oncol2019;37:2317-28. doi:10.1200/JCO.19.01009

10. Oza AM, Cook AD, Pfisterer J, Embleton A, Ledermann JA, Pujade-Lauraine E et al. Standard chemotherapy with or without bevacizumab for women with newly diagnosed ovarian cancer (ICON7): overall survival results of a phase 3 randomised trial. Lancet Oncol 2015;16:928-36. doi:10.1016/S14702045(15)00086-8

11. Aghajanian C, Goff B, Nycum LR, Wang YV, Husain A, Blank SV. Final overall survival and safety analysis of OCEANS, a phase 3 trial of chemotherapy with or without bevacizumab in patients with platinum-sensitive recurrent ovarian cancer. Gynecol Oncol2015;139:10-6.

12. Coleman RL, Brady MF, Herzog TJ, Sabbatini P, Armstrong DK, Walker JL et al. Bevacizumab and paclitaxel-carboplatin chemotherapy and secondary cytoreduction in recurrent, platinum-sensitive ovarian cancer (NRG Oncology/Gynecologic Oncology Group study GOG-0213): a multicentre, open- 
label, randomised, phase 3 trial. Lancet Oncol 2017;18:779-91. doi:10.1016/S1470-2045(17)30279-6

13. Pujade-Lauraine E, Hilpert F, Weber B, Reuss A, Poveda A, Kristensen G et al. Bevacizumab combined with chemotherapy for platinum-resistant recurrent ovarian cancer: The AURELIA open-label randomized phase III trial [published correction appears in J Clin Oncol. 2014 Dec 10;32(35):4025]. J Clin Oncol 2014;32:1302-8. doi:10.1200/JCO.2013.51.4489

14. Pignata S, Lorusso D, Joly F, Gallo C, Colombo N, Sessa C et al. Chemotherapy plus or minus bevacizumab for platinum-sensitive ovarian cancer patients recurring after a bevacizumab containing first line treatment: The randomized phase 3 trial MITO16B-MaNGO OV2B-ENGOT OV17.J Clin Oncol 2018;36, 15 suppl:5506.

15. An observational study of Avastin (bevacizumab) in combination with carboplatin/paclitaxel in first line in patients with advanced epithelial ovarian, fallopian tube or primary peritoneal cancer (OTILIA) (ClinicalTrials.gov Identifier: NCT01697488)

16. Hall M, Bertelli G, Li L, Green C, Chan S, Yeoh CC et al. Role of front-line bevacizumab in advanced ovarian cancer: the OSCAR study [published online ahead of print, 27 November 2019]. Int J Gynecol Cancer 2019, ijgc-2019-000512. doi:10.1136/ijgc-2019-000512

17. Komiyama S, Kato K, Inokuchi Y, Takano H, Matsumoto T, Hongo A et al. Bevacizumab combined with platinum-taxane chemotherapy as first-line treatment for advanced ovarian cancer: a prospective observational study of safety and efficacy in Japanese patients (JGOG3022 trial). Int J Clin Oncol 2019; 24:103-14. doi: 10.1007/s10147-018-1319-y.

18. Fuh KC, Secord AA, Bevis KS, Huh W, ElNaggar A, Blansit K et al. Comparison of bevacizumab alone or with chemotherapy in recurrent ovarian cancer patients. Gynecol Oncol 2015;139:413-8. doi:10.1016/j.ygyno.2015.06.041

19. Previs RA, Spinosa D, Fellman BM, Lorenzo A, Mulder I, Mahmound M et al. Bevacizumab beyond progression: Impact of subsequent bevacizumab retreatment in patients with ovarian, fallopian tube, and peritoneal cancer after progression. J Clin Oncol 2019;37,15 suppl: 5557

20. Lee JY, Park JY, Park SY, Lee JW, Kim JW, Kim YB et al. Real-world effectiveness of bevacizumab based on AURELIA in platinum-resistant recurrent ovarian cancer (REBECA): A Korean Gynecologic Oncology Group study (KGOG 3041). Gynecol Oncol 2019;152:61-7. doi:10.1016/j.ygyno.2018.10.031

21. Stuart GC, Kitchener H, Bacon M, duBois A, Friedlander M, Ledermann J et al. 2010 Gynecologic Cancer InterGroup (GCIG) consensus statement on clinical trials in ovarian cancer: report from the Fourth Ovarian Cancer Consensus Conference. Int J Gynecol Cancer 2011;21:750-5. doi:10.1097/IGC.0b013e31821b2568

22. Huang YF, Kuo MT, Liu YS, Cheng YM, Wu PY, Chou CY. A dose escalation study of trientine plus carboplatin and pegylated liposomal doxorubicin in women with platinum-resistant/-refractory or partially platinum-sensitive epithelial ovarian, tubal and peritoneal cancer.Front Oncol 2019;9:437. doi: 10.3389 /fonc. 2019.00437

23. Mirza MR, Monk BJ, Herrstedt J, Oza AM, Mahner S, Redondo A et al. Niraparib Maintenance Therapy in Platinum-Sensitive, Recurrent Ovarian Cancer. N Engl J Med 2016;375:2154-64. doi:10.1056/NEJMoa1611310

24. Coleman RL, Oza AM, Lorusso D, Aghajanian C, Oaknin A, Dean A et al. Rucaparib maintenance treatment for recurrent ovarian carcinoma after response to platinum therapy (ARIEL3): a randomised, double-blind, placebo-controlled, phase 3 trial [published correction appears in Lancet. 2017 Oct 28;390(10106):1948]. Lancet2017;390:1949-61. doi:10.1016/S0140-6736(17)32440-6

25. Pujade-Lauraine E, Ledermann JA, Selle F, Gebski V, Penson RT, Oza AM et al. Olaparib tablets as maintenance therapy in patients with platinum-sensitive, relapsed ovarian cancer and a BRCA1/2 mutation (SOLO2/ENGOT-Ov21): a double-blind, randomised, placebo-controlled, phase 3 trial [published correction appears in Lancet Oncol. 2017 Sep;18(9):e510]. Lancet Oncol 2017;18:1274-84. doi:10.1016/S1470-2045(17)30469-2.

26. Ray-Coquard I, Pautier P, Pignata S, Perol D, Gonzalez-Martin A, Berger R et al. Olaparib plus Bevacizumab as First-Line Maintenance in Ovarian Cancer. N Engl $J$ Med 2019;381:2416-28. doi:10.1056/NEJMoa1911361 
27. Wu YH, Chang TH, Huang YF, Chen CC, Chou CY. COL11A1 confers chemoresistance on ovarian cancer cells through the activation of Akt/c/EBP $\beta$ pathway and PDK1 stabilization. Oncotarget2015;6:23748-63. doi:10.18632/oncotarget.4250

28. Wu YH, Huang YF, Chang TH, Chou CY. Activation of TWIST1 by COL11A1 promotes chemoresistance and inhibits apoptosis in ovarian cancer cells by modulating NF- $\chi \mathrm{B}$-mediated IKK $\beta$ expression. Int J Cancer 2017;141:2305-17. doi:10.1002/ijc.30932

29. Hsu KF, Shen MR, Huang YF, Cheng YM, Lin SH, Chow NH et al. Overexpression of the RNAbinding proteins Lin28B and IGF2BP3 (IMP3) is associated with chemoresistance and poor disease outcome in ovarian cancer. Br J Cancer 2015;113:414-24. doi:10.1038/bjc.2015.254

30. Chen WC, Qiu JT, Lai CH, Huang HJ, Lin CT, Chen MY et al. Outcomes and prognoses of patients with ovarian cancer using bevacizumab: 6-year experience in a tertiary care hospital of northern Taiwan. PLoS One 2017;12:e0175703. doi:10.1371/journal.pone.0175703

31. Chiang YC, Chen CA, Chiang CJ, Hsu TH, Lin MC, You SL et al. Trends in incidence and survival outcome of epithelial ovarian cancer: 30-year national population-based registry in Taiwan. J Gynecol Oncol2013;24:342-51. doi:10.3802/jgo.2013.24.4.342

32. Lee SP, Hsu HC, Tai YJ, Chen YL, Chiang YC, Chen CA et al. Bevacizumab Dose Affects the Severity of Adverse Events in Gynecologic Malignancies. Front Pharmacol 2019;10:426. doi:10.3389/fphar.2019.00426

\section{Hosted file}

Table 1 frontline final.docx available at https://authorea.com/users/302707/articles/432802real-world-study-of-adding-bevacizumab-to-chemotherapy-for-ovarian-tubal-and-peritonealcancer-as-front-line-or-relapse-therapy-robot-an-observational-cohort-study

\section{Hosted file}

Table 2 HR final.docx available at https://authorea.com/users/302707/articles/432802-realworld-study-of-adding-bevacizumab-to-chemotherapy-for-ovarian-tubal-and-peritonealcancer-as-front-line-or-relapse-therapy-robot-an-observational-cohort-study

\section{Hosted file}

Fig 1.jpg available at https://authorea.com/users/302707/articles/432802-real-world-studyof-adding-bevacizumab-to-chemotherapy-for-ovarian-tubal-and-peritoneal-cancer-as-frontline-or-relapse-therapy-robot-an-observational-cohort-study

\section{Hosted file}

Fig 2.jpg available at https://authorea.com/users/302707/articles/432802-real-world-studyof-adding-bevacizumab-to-chemotherapy-for-ovarian-tubal-and-peritoneal-cancer-as-frontline-or-relapse-therapy-robot-an-observational-cohort-study 